\title{
Dynamical system analysis for a nonminimal torsion-matter coupled gravity
}

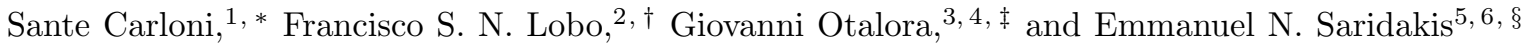 \\ ${ }^{1}$ Centro Multidisciplinar de Astrofisica - CENTRA, Instituto Superior Tecnico - IST, \\ Universidade de Lisboa - UL, Avenida Rovisco Pais 1, 1049-001, Portugal \\ ${ }^{2}$ Instituto de Astrofísica e Ciências do Espaço, Faculdade de Ciências da Universidade de Lisboa, \\ Edifício C8, Campo Grande, P-1749-016 Lisbon, Portugal \\ ${ }^{3}$ Departamento de Matemática, ICE, Universidade Federal de Juiz de Fora, Minas Gerais, Brazil \\ ${ }^{4}$ Instituto de Física Teórica, UNESP-Universidade Estadual Paulista Caixa Postal 70532-2, 01156-970, São Paulo, Brazil \\ ${ }^{5}$ CASPER, Physics Department, Baylor University, Waco, TX 76798-7310, USA \\ ${ }^{6}$ Instituto de Física, Pontificia Universidad de Católica de Valparaíso, Casilla 4950, Valparaíso, Chile
}

(Dated: December 29, 2015)

\begin{abstract}
In this work, we perform a detailed dynamical analysis for the cosmological applications of a nonminimal torsion-matter coupled gravity. Two alternative formalisms are proposed, which enables one to choose between the easier approach for a given problem, and furthermore, we analyze six specific models. In general, we extract fixed points corresponding either to dark-matter dominated, scaling decelerated solutions, or to dark-energy dominated accelerated solutions. Additionally, we find that there is a small parameter region in which the model can experience the transition from matter epoch to dark-energy era. These feature are in agreement with the observed universe evolution, and make the theory a successful candidate for the description of Nature.
\end{abstract}

PACS numbers: 04.50.Kd, 98.80.-k, 95.36.+x

\section{INTRODUCTION}

The late-time accelerated expansion of the universe is a major challenge to present-day cosmology. The converging observational evidence comes from a diverse set of cosmological data which includes observations of type Ia supernovae (SNeIa) 1], cosmic microwave background radiation $(\mathrm{CMB})[2$, constraints from SDSS galaxy clustering [3, baryon acoustic oscillations (BAO) [4] and weak lensing [5]. A plethora of theories have been proposed to explain this late-time cosmic acceleration. The simplest explanation is provided by a cosmological constant, however, this scenario is plagued by a severe fine-tuning problem associated with its energy scale [6]. Hence one has two main directions in order to seek for an alternative explanation, namely, either to introduce the concept of an exotic repulsive cosmic fluid denoted by "dark energy" in the framework of general relativity 6, 7, or to modify the gravitational sector itself 8, 9. All of these modifications to the Einstein-Hilbert action are based on the curvature description of gravity. However, an interesting and rich class of modified gravity can arise if one modifies the action of the equivalent torsional formulation of general relativity, known as "Teleparallel Equivalent of General Relativity" (TEGR) [10 13. Thus, if one desires to modify gravity starting from this formulation, the simplest model is to extend the torsion scalar $T$ in the Lagrangian to an arbitrary function $f(T)$ 14 19] (for a review see 20]).

\footnotetext{
* sante.carloni@tecnico.ulisboa.pt

$\dagger$ fslobo@fc.ul.pt

$\ddagger$ giovanni@ift.unesp.br

$\S$ Emmanuel_Saridakis@baylor.edu
}

Recently, an extension of $f(T)$ gravity was proposed in which the torsion scalar is coupled non-minimally to the matter sector 21. This theory is a novel gravitational modification, since it is different from both $f(T)$ gravity, as well as from the original nonminimal curvaturematter-coupled theory [22]. Additionally, the cosmological applications of this new theory proves to be very interesting [21]. More specifically, a wide variety of cosmological scenarios were obtained, such as an effective dark energy sector whose equation-of-state parameter can be quintessence or phantom-like, or exhibit the phantomdivide crossing, while for a large range of the model parameters the Universe results in a de Sitter, dark-energydominated, accelerating phase. Furthermore, early-time inflationary solutions were also obtained, and thus these models provide a unified description of the cosmological history. It is interesting to note, that along these lines of research, another extension of $f(T)$ gravity was also proposed [23] (motivated by Ref. 24]), that allowed for a general coupling of the torsion scalar $T$ with the trace of the matter energy-momentum tensor $\mathcal{T}$. The resulting $f(T, \mathcal{T})$ theory also possesses interesting cosmological phenomenology, leading to a unified description of the initial inflationary phase, the subsequent nonaccelerating, matter-dominated expansion, and then the transition to a late-time accelerating phase. Additionally, a detailed study of the scalar perturbations at the linear level revealed that $f(T, \mathcal{T})$ cosmology may be free of ghosts and instabilities for a wide class of the model parameters.

In the present paper, we are interested in performing a detailed phase space analysis of the non-minimal torsion-matter coupling, and explore the phenomenology associated to some specific models. In particular, we apply the Dynamical System Approach, developed amongst 
others by Ellis and Wainwright 25, 26. This approach is able to by-pass the non-linearities of the cosmological equations, that forbid an analytical treatment, and allow for the extraction of the general features and global behavior of the evolution, independently of the specific initial conditions. Using this technique we will be able to show that this class of models often present a matterdominated epoch and that providing suitable forms for the functions of torsion, that appear in the theory, the universe evolves towards a late-time acceleration.

The manuscript is organised in the following manner: In Section, II we briefly review the scenario of nonminimally torsion-matter coupled gravity and provide the corresponding cosmological equations. In Section, III we perform the dynamical analysis of this class of cosmologies, focusing on three different models. In Section [V] for completeness, we present an alternative approach to the problem, which offers complementary information in relation to the first formulation. Finally, Section $\mathrm{V}$ is devoted to the conclusions and discussion.

\section{THE MODEL}

The action of non-minimally torsion-matter coupled gravity can be written in the following manner [21]

$$
S=\int d^{4} x e\left[\kappa f_{1}(T)-f_{2}(T) \mathcal{L}_{m}\right]
$$

where $\kappa=1 / 16 \pi G$ and $f_{i}(T)$ (with $i=1,2$ ) are arbitrary functions of the torsion scalar $T$. The latter is defined as

$$
T \equiv S_{\rho}{ }^{\mu \nu} T_{\mu \nu}^{\rho}=\frac{1}{4} T_{\rho}{ }^{\mu \nu} T_{\mu \nu}^{\rho}+\frac{1}{2} T_{\nu}^{\rho \mu} T_{\mu \rho}^{\nu}-T_{\rho \mu}{ }^{\rho} T_{\nu}^{\nu \mu},
$$

where

$$
\begin{array}{r}
T_{\mu \nu}^{\rho} \equiv e_{A}^{\rho}\left(\partial_{\mu} e_{\nu}^{A}-\partial_{\nu} e_{\mu}^{A}\right), \\
K_{\rho}^{\mu \nu} \equiv-\frac{1}{2}\left(T_{\rho}^{\mu \nu}-T_{\rho}^{\nu \mu}-T_{\rho}^{\mu \nu}\right), \\
S_{\rho}^{\mu \nu} \equiv \frac{1}{2}\left(K_{\rho}^{\mu \nu}+\delta_{\rho}^{\mu} T_{\theta}^{\theta \nu}-\delta_{\rho}^{\nu} T_{\theta}^{\theta \mu}\right),
\end{array}
$$

are respectively the torsion tensor, the contortion tensor and the "superpotential" 20].

Varying the action with respect to the tetrad $e_{\rho}^{A}$ yields the field equations

$$
\begin{array}{r}
e^{-1} \partial_{\nu}\left(e F e_{A}^{\rho} S_{\rho}^{\mu \nu}\right)+F e_{A}^{\tau} T_{\tau \nu}^{\rho} S_{\rho}^{\nu \mu}+\frac{1}{4} \kappa f_{1} e_{A}^{\mu} \\
+\kappa f_{2}^{\prime} \partial_{\nu} T e_{A}^{\tau}{ }_{S^{\mathrm{em}}}^{\mu \nu}=\frac{1}{4} f_{2} e_{A}^{\nu} T_{\nu}^{\mathrm{em}}{ }_{\nu}^{\mu}
\end{array}
$$

where we have defined

$$
\stackrel{\mathrm{em}}{S}_{A}^{\rho \mu}=\frac{1}{4 \kappa} \frac{\partial \mathcal{L}_{m}}{\partial \partial_{\mu} e_{\rho}^{A}},
$$

and $F \equiv \kappa f_{1}^{\prime}-f_{2}^{\prime} \mathcal{L}_{m}$, with the prime denoting differentiation with respect to the torsion scalar. The matter energy-momentum tensor is defined as

$$
\stackrel{\mathrm{em}}{T}{ }_{A}^{\rho}=\frac{1}{e} \frac{\delta S_{m}}{\delta e_{\rho}^{A}}
$$

In a purely space-time form, the Bianchi identities of Teleparallel Gravity imply the relationship

$$
\bar{\nabla}_{\mu}{ }_{T}^{\mathrm{em}}{ }_{\tau}^{\mu}=\frac{4}{f_{2}} K_{\mu \tau}^{\rho} S_{\rho}^{\mu \nu} \bar{\nabla}_{\nu} F-\frac{f_{2}^{\prime}}{f_{2}}\left(\stackrel{\mathrm{em}}{T}_{\tau}{ }^{\mu}-\mathcal{L}_{m} \delta_{\tau}^{\mu}\right) \bar{\nabla}_{\mu} T,
$$

where $\bar{\nabla}_{\mu}$ is the covariant derivative in the Levi-Civita connection [11]. Additionally, we have assumed that the matter Lagrangian $\mathcal{L}_{m}$, depends only on the tetrad and not on its derivatives. Thus, the coupling between the matter and torsion describes an exchange of energy and momentum between both.

In order to obtain a cosmological model governed by $f(T)$ gravity, we have to impose the usual homogeneity and isotropy conditions. Therefore, we consider the common choice for the tetrad field, given by

$$
e_{\mu}^{A}=\operatorname{diag}(1, a(t), a(t), a(t)),
$$

which corresponds to a flat Friedmann-Robertson-Walker (FRW) background, with $a(t)$ the scale factor. Since the Lagrangian density of a perfect fluid is the energy scalar, representing the energy in a local rest frame for the fluid, a possible "natural choice" for the matter Lagrangian density is $\mathcal{L}_{m}=\rho_{m}$ 27. However, we mention that in the presence of non-minimal couplings in which the matter Lagrangian appears explicitly in the gravitational field equations, special care should be taken (we refer the reader to [28 for more details). Nevertheless, throughout this work we consider $\mathcal{L}_{m}=\rho_{m}$. In this case we have ${ }_{S}^{\mathrm{em}}{ }_{A} \rho \mu=0$, and also the usual form of the energy-momentum tensor for the perfect fluid is given by

$$
\stackrel{\mathrm{em}}{T}_{\mu \nu}=\left(\rho_{m}+p_{m}\right) u_{\mu} u_{\nu}-p_{m} g_{\mu \nu} .
$$

One can see that the energy-momentum tensor is again conserved, just like in teleparallel gravity or $f(T)$ theories, since Eq. (7) yields the continuity equation

$$
\dot{\rho}_{m}+3 H\left(1+\omega_{m}\right) \rho_{m}=0,
$$

where $H=\dot{a} / a$ is the Hubble parameter and $\omega_{m} \equiv$ $p_{m} / \rho_{m}$ is the equation-of-state (EOS) parameter of matter. In the above expressions the dot represents a derivatives with respect to cosmic time.

Inserting the flat FRW vierbein choice (8) into the field equations (4) we obtain the modified Friedmann equations 21]

$$
\begin{aligned}
12 F H^{2} & =f_{2} \rho_{m}-\kappa f_{1}, \\
\dot{F} H+F \dot{H} & =-\frac{1}{4} \gamma f_{2} \rho_{m},
\end{aligned}
$$

where we have defined $\gamma \equiv 1+\omega_{m}$. Note that a useful relation arising from the choice (8) of the tetrad is that 
the torsion scalar is proportional to the square of the Hubble parameter, namely $T=-6 H^{2}$.

The generalized Friedmann equations can be rewritten in the standard form

$$
\begin{aligned}
3 H^{2} & =\frac{1}{2 \kappa}\left(\rho_{D E}+\rho_{m}\right), \\
2 \dot{H} & =-\frac{1}{2 \kappa}\left(\rho_{m}+p_{m}+\rho_{D E}+p_{D E}\right),
\end{aligned}
$$

where the effective energy density and effective pressure of the dark energy sector are defined as

$$
\begin{gathered}
\rho_{D E}=\left(\frac{\kappa f_{2}}{2 F}-1\right) \rho_{m}-\frac{\kappa^{2} f_{1}}{2 F}, \\
p_{D E}=\left(\frac{\kappa f_{2}}{F}-1\right) \gamma \rho_{m}+\frac{4 \kappa H \dot{F}}{F}-\rho_{D E},
\end{gathered}
$$

respectively. One can easily verify that the dark energy density and pressure satisfy the continuity equation

$$
\dot{\rho}_{D E}+3 H\left(1+\omega_{D E}\right) \rho_{D E}=0,
$$

in accordance with Eqs. (7) and (10). We have defined the dark-energy equation-of-state parameter as usual, namely, $\omega_{D E} \equiv p_{D E} / \rho_{D E}$.

\section{DYNAMICAL SYSTEM ANALYSIS}

In order to perform the phase-space analysis of the cosmological scenario at hand, we have to introduce suitable dimensionless auxiliary variables that will bring the system of cosmological equations into its autonomous form [25, 26]. We choose

$$
X=\frac{f_{1}}{12 H^{2} f_{1}^{\prime}}, \quad Y=\frac{f_{2}}{12 H^{2} f_{2}^{\prime}}, \quad \Omega=\frac{\rho_{m}}{6 H^{2} \kappa},
$$

and the logarithmic time $N=\log a$. With this choice, and taking in account that $T=-6 H^{2}$, the case of a standard $f(T)$ theory is recovered when $|Y| \rightarrow \infty$.

Using the variables 18 the cosmological equations are equivalent to the autonomous system

$$
\begin{aligned}
& \frac{d X}{d N}=-\frac{3 \gamma \mathbf{Q}(X+1)(Y+1)(2(\mathbf{W}+1) X+1)}{\mathbf{P}(X+1)-\mathbf{Q}(2 \mathbf{W}(Y+1)-X+Y)}, \\
& \frac{d Y}{d N}=-\frac{3 \gamma(X+1)(Y+1)(\mathbf{P} Y+2 \mathbf{Q} Y+\mathbf{Q})}{\mathbf{P}(X+1)-\mathbf{Q}(2 \mathbf{W}(Y+1)-X+Y)}, \\
& 2 \mathbf{Q}(Y+1) \Omega+X+1=0,
\end{aligned}
$$

with $\gamma \equiv 1+\omega_{m}$, as defined above, and where

$$
\mathbf{Q}=\frac{T f_{2}^{\prime}}{2 f_{1}^{\prime}}, \quad \mathbf{P}=\frac{T^{2} f_{2}^{\prime \prime}}{f_{1}^{\prime}}, \quad \mathbf{W}=\frac{T f_{1}^{\prime \prime}}{f_{1}^{\prime}},
$$

are functions only of $T$. Their expression in terms of the variables 18 can be obtained noting that

$$
\frac{X}{Y}=\frac{f_{1} f_{2}^{\prime}}{f_{2} f_{1}^{\prime}} .
$$

Inverting the above equation for a given form of $f_{1}$ and $f_{2}$, one obtains $T=T(X / Y)$ and the system $\sqrt{19}$ can be closed. It is important to stress at this point that since $T=-6 H^{2}$ must be non positive, the requirement that $T(X / Y) \leq 0$ implies that only some parts of the phase space defined by those variables will have actual physical meaning. In fact, this restriction implies that even if some physically interesting fixed points are present there might not be physical orbits that connect them. Additionally, note that since we have used the third equation of $(19)$ to eliminate $\Omega$, we might need to discard the $Y=-1$ part of the phase space.

Using the dimensionless variables from Eqs. 18 and (20), the dark-energy density parameter is written as

$$
\Omega_{D E} \equiv \frac{\rho_{D E}}{6 \kappa H^{2}}=1-\Omega
$$

Due to the definition (18) the physically relevant part of the phase space will necessarily have $\Omega \geq 0$, however, since $\Omega_{D E}$ does not need to be defined positive, $\Omega$ is not necessarily less than one. In the following however, we will require, at least for the matter-dominated fixed points, that $0<\Omega<1$ and therefore that $0<\Omega_{D E}<1$.

The solutions associated to the fixed points can be obtained using the equation

$$
\begin{aligned}
& \frac{\dot{H}}{H^{2}}=-\frac{1}{p}, \\
& \frac{1}{p}=-\frac{3 \gamma \mathbf{Q}_{*}\left(X_{*}+1\right)\left(Y_{*}+1\right)}{\mathbf{P}_{*}\left(X_{*}+1\right)-\mathbf{Q}_{*}\left(2 \mathbf{W}_{*}\left(Y_{*}+1\right)-X_{*}+Y_{*}\right)},
\end{aligned}
$$

where $p$ is a constant and an asterisk represents the value of a variable at the fixed point $\left\{X_{*}, Y_{*}\right\}$. Hence, the solutions obtained in this way are all scaling solutions which can alleviate the coincidence problem [6].

The parameter of deceleration is defined as

$$
q \equiv-\frac{\dot{H}}{H^{2}}-1=\frac{1}{p}-1 .
$$

Thus, when $H=H_{0}=$ constant (and therefore $q=-1$ ) we have the de Sitter solution $a(t) \propto e^{H_{0} t}$ and accelerated expansion for all values. For finite $p>0$ we acquire $a(t) \propto t^{p}$, and we have an accelerated solution if $p>1$ $(q<0)$ or a decelerated solution if $0<p<1(q>0)$.

In the following subsections we apply the above general technique to three specific cosmological models, obtained by specific choices of the functions $f_{1}$ and $f_{2}$.

A. Model I: $f_{1}(T)=T+\alpha T^{2}-\Lambda$ and $f_{2}(T)=1+\beta T$

As a first example we consider the specific choices, given by

$$
f_{1}(T)=T+\alpha T^{2}-\Lambda, \text { and } f_{2}(T)=1+\beta T,
$$


with $\alpha, \beta$ and $\Lambda$ constants. In this case, the auxiliary variable choice 18 implies

$$
\frac{X}{Y}=\frac{\beta\left(-\Lambda+\alpha T^{2}+T\right)}{(2 \alpha T+1)(\beta T+1)},
$$

and

$$
T(X, Y)=\frac{\beta Y-(2 \alpha+\beta) X+K}{2 \alpha \beta(2 X-Y)},
$$$$
K= \pm \sqrt{[(2 \alpha+\beta) X-\beta Y]^{2}-4 \alpha \beta(2 X-Y)(X+\beta \Lambda Y)} .
$$

Therefore, for these choices of the functions $f_{1}$ and $f_{2}$, we have two possible solutions for $T(X / Y)$. In the following, we will choose the solution with positive $K$. Inserting the functions in Eq. 201, one has

$$
\mathbf{Q}=\frac{\beta T}{2(1+2 \alpha T)}, \quad \mathbf{P}=0, \quad \mathbf{W}=\frac{2 \alpha T}{2 \alpha T+1} .
$$

We will analyse the autonomous system and the fixed points, and their respective stability below.

\section{Autonomous system and fixed points}

Substituting the expressions for $\mathbf{Q}, \mathbf{P}$ and $\mathbf{W}$ into the dynamical system 19 we find

$$
\begin{aligned}
& \frac{d X}{d N}=-\frac{3 \gamma(X+1)(Y+1)[2 \alpha T+2 X(4 \alpha T+1)+1]}{2 \alpha T(X-3 Y-2)+X-Y} \\
& \frac{d Y}{d N}=-\frac{3 \gamma(X+1)(Y+1)(2 Y+1)(2 \alpha T+1)}{2 \alpha T(X-3 Y-2)+X-Y}
\end{aligned}
$$

where $T$ is given by (27). The dynamical system presents the invariant submanidfolds $X=-1, Y=-1, Y=$ $-1 / 2, T=-1 / 2 \alpha$. Some of these submanifolds, such as $X=-1$, represent fixed points, whereas others can be singular, as in the case of $Y=-1$. The presence of invariant submanifolds additionally implies that the phase space does not admit a global attractor.

The critical points are obtained setting $d X / d N=$ $d Y / d N=0$ and are shown in Table I. They consist of a line of fixed points $\mathcal{A}$ characterized by $X=-1$, associated via 23 to the solution $a(t) \propto e^{H_{0} t}$ with

$$
H_{0}=\frac{1}{6} \sqrt{\frac{1+\sqrt{1-12 \alpha \Lambda}}{\alpha}},
$$

and $\Omega \propto \rho_{m}=0$, i.e., they correspond to a dark-energy dominated, de Sitter universe.

In addition to the line $\mathcal{A}$, the phase space admits the couple of fixed points $\mathcal{B}$ for $Y=-1 / 2$. They are associated to the solution $a(t) \propto t^{p}$ with $H=p / t$ and

$$
p= \pm \frac{4 \alpha}{3 \gamma} \sqrt{\frac{\beta \Lambda+1}{\alpha \beta(4 \alpha \Lambda+1)}},
$$

where the \pm signs guarantee that $p>0$.

Points $\mathcal{B}$ exist only if the inequality $\alpha X_{\mathcal{B}}<0$ is satisfied, and they correspond to physical states only if the torsion scalar evaluated in their coordinates is negative. The same happens with the line $\mathcal{A}$ : in general, only segments of it will be in the physical $T<0$ part of the phase space. These complex constraints make a complete analysis of this example, which are too complicated to be fully included here. Hence, in order to highlight physically interesting scenarios we will choose parameter values such that at least one of the points $\mathcal{B}$ is in the $T<0,1<\Omega<0$ region of the phase space. One set of parameter values for which this is possible is

$$
\begin{aligned}
& \alpha>0, \quad \beta>2 \alpha, \quad \frac{\beta-16 \alpha}{12 \alpha \beta}<\Lambda<\Lambda_{*}, \\
& \Lambda_{*}=-\frac{64 \alpha^{3}+24 \alpha^{2} \beta+\beta^{3}}{32 \alpha^{2} \beta(3 \alpha+\beta)}-\frac{\sqrt{16 \alpha^{2}+\beta^{2}}}{32 \alpha^{2}}\left|\frac{\beta^{2}-16 \alpha^{2}}{\beta(3 \alpha+\beta)}\right| .
\end{aligned}
$$

With these choices, $\mathcal{A}$ satisfies the constraint on $T<0$ for

$$
\frac{1}{\beta \Lambda}<Y \leq 2 \sqrt{\frac{-\alpha^{2}+\alpha \beta^{2} \Lambda+\alpha \beta}{\beta^{2}(4 \alpha \Lambda+1)}}-1
$$

and moreover only one point $\mathcal{B}$ exists for which $0<\Omega<1$ and $0<p<1$.

\section{Stability of fixed points}

The stability of the fixed points is deduced using the Hartmann-Grobmann theorem [29, and the stability properties for each critical point are shown in Table I. The de Sitter line $\mathcal{A}$ is always attractive with eigenvalues

$$
\mu_{1}=0, \quad \mu_{2}=-3 \gamma
$$

Here the zero eigenvalue is related to the fact that we are dealing with a line of fixed points rather than with a nonhyperbolic character of the point. The character of the line is determined by the sign on the non-zero eigenvalue and is therefore attractive.

On the other hand, the eigenvalues of the critical points $\mathcal{B}$ are given by

$$
\mu_{1}=\frac{3}{2} \gamma \sqrt{\frac{\beta(4 \alpha \Lambda+1)}{\alpha(\beta \Lambda+1)}}, \quad \mu_{2}=3 \gamma\left(2-\frac{\beta}{2 \alpha}\right) .
$$

It follows that point $\mathcal{B}$ can only be a saddle in the parameter range we consider. Choosing suitable initial conditions, it is therefore possible that the model will present an unstable phase of decelerated expansion followed by accelerated expansion. An example of this case is shown in the phase-space portrait of Fig. 1, arising through a numerical elaboration in the case of $\alpha=1, \beta=1, \Lambda=-6 / 5$. The phase space presented also 


\begin{tabular}{|c|c|c|c|c|c|c|}
\hline Name & $\left\{X_{*}, Y_{*}\right\}$ & $\overline{a(t)}$ & $\rho(t)$ & $\Omega_{D E}=1-\Omega$ & Deceleration parameter $(q)$ & Stability \\
\hline $\mathcal{A}$ & $\left\{-1, Y_{*}\right\}$ & $e^{H_{0} t}$ & 0 & 1 & -1 & Attractors \\
\hline $\mathcal{B}$ & $\left\{X_{0},-\frac{1}{2}\right\}$ & $t^{p}$ & $t^{-3 \gamma p}$ & $1-\frac{16 \alpha X_{0}\left(X_{0}+1\right)}{\beta+2 X_{0}(2 \alpha+\beta)-4 \alpha X_{0}}$ & $\frac{1}{p}-1$ & Saddle \\
\hline$H_{0}$ & $\frac{1}{6} \sqrt{\frac{1+\sqrt{1-}}{\alpha}}$ & $\overline{\overline{12 \alpha \Lambda}}$, & $X_{0}$ & $\frac{4 \alpha \beta \Lambda+\beta-2 \sqrt{4 \alpha^{2} \beta^{2} \Lambda^{2}+4 \alpha}}{2(4 \alpha-\beta)}$ & $\overline{\chi^{2} \beta \Lambda+\alpha \beta^{2} \Lambda+\alpha \beta}$, & $\frac{\beta \Lambda+1}{\alpha \beta(4 \alpha \Lambda+1)}$ \\
\hline
\end{tabular}

Table I. The critical points for Model I. Here $Y_{*}$ are the values of Y for which $T=-6 H^{2}<0$.

reveals that there are orbits which evolve towards the singularity of the system. This could deduce an instability for this class of models, and therefore it restricts the range of viable initial conditions.

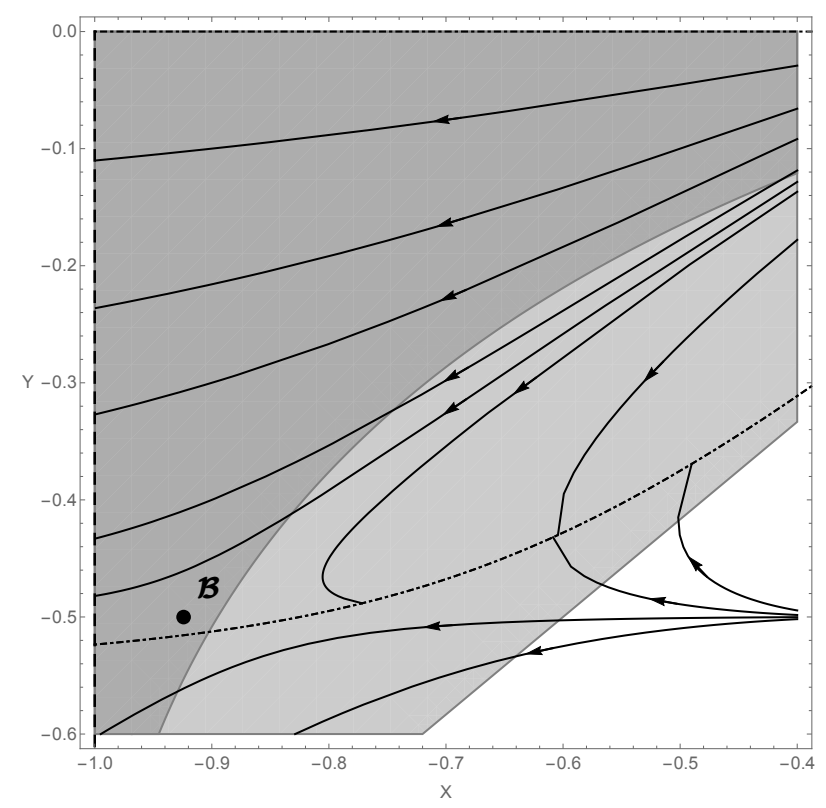

FIG. 1. An example of the phase space for Model $I: f_{1}(T)=$ $T+\alpha T^{2}-\Lambda$ and $f_{2}(T)=1+\beta T$, with $\alpha=1, \beta=1, \Lambda=-6 / 5$. The dark grey area is the part of the phase space for which $T<0$ and the light gray one is the one in which $0<\Omega<1$. The dashed line represents the line of fixed points $\mathcal{A}$. The dotdashed lines represent the value of the phase space for which the dynamical equations are singular.

B. Model II: $f_{1}(T)=T-\Lambda$ and $f_{2}(T)=1-\alpha T+\beta T^{2}$

We consider now the case

$$
f_{1}(T)=T-\Lambda, \text { and } f_{2}(T)=1-\alpha T+\beta T^{2},
$$

where $\alpha, \beta$ and $\Lambda$ are constants. For this model we have that

$$
\frac{X}{Y}=\frac{(T-\Lambda)(2 \beta T-\alpha)}{T(\beta T-\alpha)+1}
$$

and therefore

$$
\begin{aligned}
& T(X, Y)=\frac{\alpha X-Y(\alpha+2 \beta \Lambda)}{2 \beta(X-2 Y)} \\
& K= \pm \sqrt{[\alpha X-Y(\alpha+2 \beta \Lambda)]^{2}-4 \beta(X-2 Y)(X-\alpha \Lambda Y)}
\end{aligned}
$$

Thus, similarly to the Model I above, in this case there are multiple solutions for the expression of the torsion in terms of the variables $X, Y$. In the following we will choose the expression where $K$ is positive.

The parameters $\mathbf{Q}, \mathbf{P}, \mathbf{W}$ become

$$
\mathbf{Q}=-\frac{1}{2} T(\alpha-2 \beta T), \quad \mathbf{P}=2 \beta T^{2}, \quad \mathbf{W}=0 .
$$

The conditions $T \leq 0$ and $0 \leq \Omega \leq 1$ lead to complex constraints of the allowed intervals of the variables, which depend on the values of the constants $\alpha, \beta, \Lambda$. In order to select a class of physically interesting cases, we choose

$$
\alpha>0, \quad \beta<0, \quad \frac{\alpha}{4 \beta}<\Lambda<\Lambda_{*},
$$

where $\Lambda_{*}$ is a solution of the algebraic equation

$$
\begin{aligned}
& \alpha^{5}-3 \alpha^{3} \beta-\Lambda^{2}\left(-16 \alpha^{5} \beta+112 \alpha^{3} \beta^{2}-240 \alpha \beta^{3}\right) \\
& -\Lambda\left(\alpha^{6}+12 \alpha^{4} \beta-100 \alpha^{2} \beta^{2}+192 \beta^{3}\right)-64 \beta^{4} \Lambda^{3}=0 .
\end{aligned}
$$

\section{Autonomous system and fixed points}

Substituting the expressions for $\mathbf{Q}, \mathbf{P}, \mathbf{W}$, the dynamical equations become

$$
\begin{aligned}
& \frac{d X}{d N}=-\frac{3 \gamma(X+1)(2 X+1)(Y+1)(\alpha-2 \beta T)}{\alpha(X-Y)-2 \beta T(3 X-Y+2)} \\
& \frac{d Y}{d N}=-\frac{3 \gamma(X+1)(Y+1)[(1+2 Y) \alpha-2 \beta T(4 Y+1)]}{\alpha(X-Y)-2 \beta T(3 X-Y+2)}
\end{aligned}
$$




\begin{tabular}{ccccccc}
\hline \hline Name & $\left\{X_{*}, Y_{*}\right\}$ & $a(t)$ & $\rho(t)$ & $\Omega_{D E}=1-\Omega$ & Deceleration factor $(q)$ & Stability \\
\hline $\mathcal{A}$ & $\left\{-1, Y_{*}\right\}$ & $e^{H_{0} t}$ & 0 & 1 & -1 & Attractor \\
$\mathcal{B}$ & $\left\{-\frac{1}{2}, Y_{0}\right\}$ & $t^{p}$ & $t^{-3 \gamma p}$ & $1+\frac{\beta\left(4 Y_{0}+1\right)^{2}}{2 \alpha Y_{0}\left(Y_{0}+1\right)\left(\alpha+2 \alpha Y_{0}\right)}$ & $\frac{1}{p}-1$ & Repeller \\
\hline & & $H_{0}=\sqrt{\frac{\Lambda}{6}}$, & $Y_{0}=\frac{4 \beta-\alpha^{2}-2 \sqrt{\beta\left(\alpha^{2}-4 \beta\right)(\alpha \Lambda-1)}}{2 \alpha(\alpha+4 \beta \Lambda)}, \quad p=\frac{4 \sqrt{\beta(\alpha \Lambda-1)}}{3 \gamma \sqrt{\alpha^{2}-4 \beta}}$ & \\
\hline \hline
\end{tabular}

Table II. The critical points for Model II in the case $\beta>\alpha^{2} / 4$. Here $Y_{*}$ are the values of Y for which $T=-6 H^{2}<0$.

where $T$ is given by 39 . The system is similar to the one of Mode I above, and presents the same invariant submanifolds. Setting to zero the L.H.S. of the equations we find a critical line $\mathcal{A}$ for $X=-1$. In the chosen interval for the parameters this line is physical $(T<0)$ for $\alpha<0$ and

$$
\begin{aligned}
& Y \leq Y_{1} \wedge Y>-1 / 2, \\
& Y_{1}=\frac{4 \beta-\alpha^{2}}{(\alpha+2 \beta \Lambda)^{2}}-2 \sqrt{-\frac{\beta\left(\alpha^{2}-4 \beta\right)(\Lambda(\alpha+\beta \Lambda)+1)}{(\alpha+2 \beta \Lambda)^{4}}} .
\end{aligned}
$$

Furthermore, the system admits two additional fixed points. However, in the parameter region we have considered, only one of them, named $\mathcal{B}$, lies in the $T<0$ part of the phase space.

The solutions associated to these fixed points are obtained from Eq. 23). The points on the critical line $\mathcal{A}$ satisfiy $a \sim e^{H_{0} t}$ with

$$
H_{0}=\sqrt{\frac{\Lambda}{6}}=\text { const, }
$$

and thus they represent an accelerated expansion for all parameter values, with $q=-1$ and $\Omega=0$, i.e., they correspond to a dark-energy dominated, de-Sitter universe. On the other hand, point $\mathcal{B}$ represents the power-law solutions $a \sim t^{p}$ with $H=p / t$ and

$$
p=\frac{4 \sqrt{\beta(\alpha \Lambda-1)}}{3 \gamma \sqrt{\alpha^{2}-4 \beta}} .
$$

Note that with our choice of parameters, $\alpha \Lambda-1$ is always positive, and therefore this solution always represents a decelerated solution (with $0 \leq \Omega \leq 1$ ).

\section{Stability of fixed points}

The de Sitter line $\mathcal{A}$ is always attractive with eigenvalues $\mu_{1}=0$ and $\mu_{2}=-3 \gamma$. On the other hand, for the critical point $\mathcal{B}$ the eigenvalues are given by

$$
\mu_{1}=\frac{3 \gamma \sqrt{\alpha^{2}-4 \beta}}{2 \sqrt{\beta(\alpha \Lambda-1)}}, \quad \mu_{2}=-\frac{3 \gamma(\alpha-4 \beta \Lambda)}{2 \beta \Lambda} .
$$

Hence, for the physically meaningful region $0 \leq \Omega \leq 1$ and $T \leq 0$, the critical point $\mathcal{B}$ is always a repeller and only for very specific initial conditions there are orbit that can connect $\mathcal{B}$ to the line of fixed points $\mathcal{A}$ within the physical part of the phase space. The critical points of Model II and their features, are summarized in Table II. Finally, for completeness, in Fig. 2 we provide a specific phase-space portrait, arising from a numerical analysis for the case of $\alpha=5, \beta=-18 / 5, \Lambda=-2 / 7$.

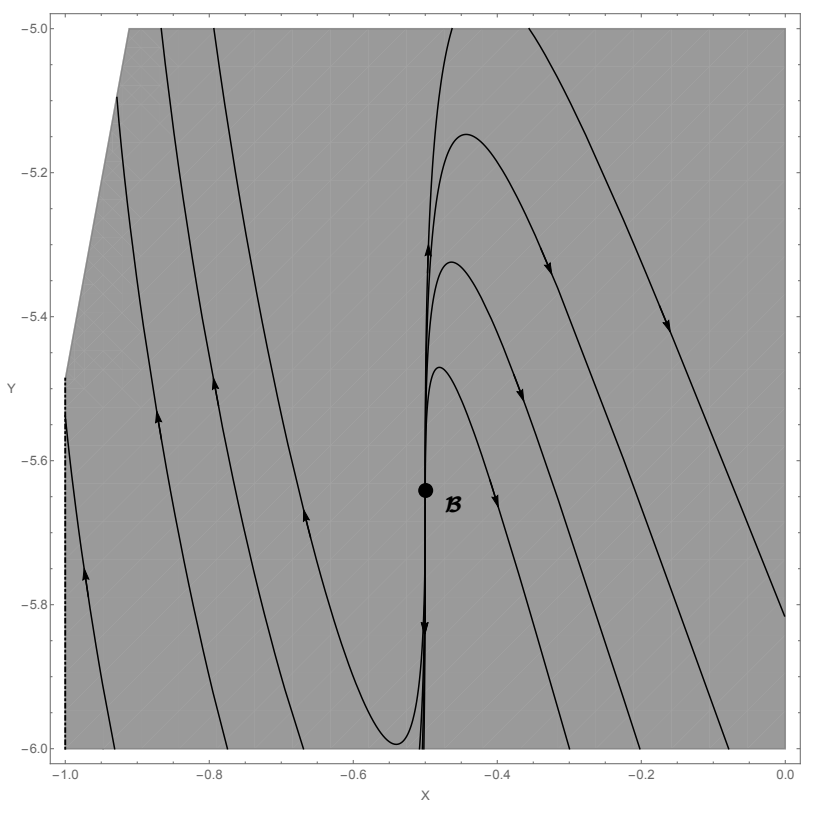

FIG. 2. An example of the phase space for Model II: $f_{1}(T)=$ $T-\Lambda$ and $f_{2}(T)=1-\alpha T+\beta T^{2}$, with $\alpha=5, \beta=-18 / 5, \Lambda=$ $-2 / 7$. The grey area is the part of the phase space for which $T<0$ and $0<\Omega<1$. The dot-dashed line represents the line of fixed points $\mathcal{A}$ that have $T<0$.

C. Model III: $f_{1}(T)=G e^{A T^{2}}$ and $f_{2}(T)=\Lambda e^{-T}$

We consider now the case with exponential couplings

$$
f_{1}(T)=G e^{A T^{2}}, \text { and } f_{2}(T)=\Lambda e^{-T},
$$


where $A, G$ and $\Lambda$ are constants.

For the form of coupling given above we have that

$$
T=-\frac{Y}{2 A X},
$$

and

$$
\begin{aligned}
& \mathbf{Q}=-\frac{\Lambda}{4 A G} e^{\frac{Y(2 X-Y)}{4 A X^{2}}}, \\
& \mathbf{P}=\frac{\Lambda Y}{4 A^{2} G X} e^{\frac{Y(2 X-Y)}{4 A X^{2}}}, \\
& \mathbf{W}=\frac{Y^{2}}{2 A X^{2}}+1 .
\end{aligned}
$$

Hence, in this case only $Y / A X>0$ represents the physical part of the phase space. In the following, for simplicity, we will only consider $\Lambda>0,0<G<3 / 7$ and $A>0$.

\section{Autonomous system and fixed points}

For this model the dynamical equations are written as

$$
\begin{aligned}
& \frac{d X}{d N}=\frac{3 \gamma X(X+1)(Y+1)\left[A X(4 X+1)+Y^{2}\right]}{A X^{2}(X-3 Y-2)+Y(X-Y)(X+Y+1)}(52) \\
& \frac{d Y}{d N}=\frac{3 \gamma X(X+1)(Y+1)\left[A(2 X Y+X)+Y^{2}\right]}{A X^{2}(2+3 Y-X)+Y(Y-X)(X+Y+1)}(53)
\end{aligned}
$$

As in the previous models, the above system presents the invariant submanifolds $X=0, X=-1$ and $Y=-1$ and their existence implies that no global attractor exists in the phase space. The submanifold $X=0$ has to be excluded as it represents a divergent torsion and in order to use our general approach one has to exclude $Y=-1$.

The system admits, therefore, a line of fixed points and an isolated fixed point. The critical line $\mathcal{A}$ is a de-Sitter fixed point such that $a \sim e^{H_{0} t}$ with

$$
H_{0}=\frac{1}{2 \sqrt{3} \sqrt[4]{A}}
$$

and corresponds to $T<0$ only if $Y_{*}<0$. The critical point $\mathcal{B}$ is a scaling solution with $X_{*} / A Y_{*}=1$. The associated solution is obtained from (23), and it represents a power-law solution $a \sim t^{p}$ with $H=p / t$ and exponent $p$ given by

$$
p=\frac{2(A+1)}{3 \gamma} .
$$

Thus, it can be either an accelerated or decelerated solution.

\section{Stability of fixed points}

As in the previous examples, using the HartmanGrobman theorem we can analyze the stability of the fixed subspaces. For the line $\mathcal{A}$ the eigenvalues are

$$
\mu_{1}=0, \quad \mu_{2}=-3 \gamma \text {. }
$$

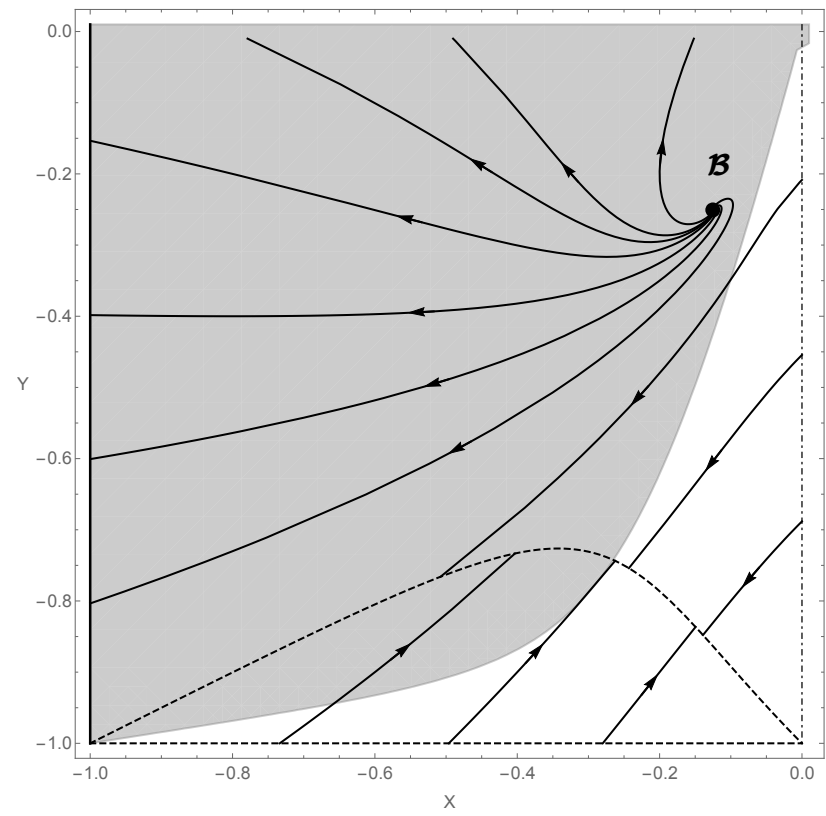

FIG. 3. An example of the phase space of Model III: $f_{1}(T)=$ $\Lambda e^{A T}$ and $f_{2}(T)=e^{\frac{A}{2 \alpha} T^{-2 \alpha}}$, with $A=1, \gamma=1, \Lambda=1, G=$ $1 / 10$. Here the grey area represents the region in which $0<$ $\Omega<1$, the balck line is the fixed line $\mathcal{A}$, the dot-dashed line in the invariant submanifold $X=0$, the dashed lines represent singularities for the dynamical system.

which is independent for $Y_{*}$. For point $\mathcal{B}$ they read

$$
\mu_{1,2}=\frac{3 \gamma(3 A \pm \sqrt{(A-8) A})}{2(A+1)}
$$

so that for $A>0$ this point can be a repeller or a saddle. The critical points of Model III and their features, are summarized in Table

It turns out that this model is able, for the interval of parameters we have considered, to describe a transition between decelerated and accelerated expansion. In Fig. 3. we present a phase-space portrait for $A=1, \gamma=1, \Lambda=$ $1, G=1 / 10$. Similarly to Model I above, in this case there also appear orbits which evolve towards singular states for the model.

\section{AN ALTERNATIVE DYNAMICAL-SYSTEMS APPROACH}

The analysis performed in the previous section does not make full use of the key relationship $T=-6 H^{2}$. In order to formulate an approach which will be optimal in this respect, let us consider the form of Eq. (11). Since $\mathcal{L}_{m}=\rho_{m}$ one has

$$
\kappa\left(12 H^{2} f_{1}^{\prime}+f_{1}\right)-\rho_{m}\left(12 H^{2} f_{2}^{\prime}+f_{2}\right)=0 .
$$




\begin{tabular}{|c|c|c|c|c|c|c|}
\hline Name & $\left\{X_{*}, Y_{*}\right\}$ & $a(t)$ & $\rho(t)$ & $\Omega_{D E}=1-\Omega$ & leration factor $(q$ & Stability \\
\hline $\mathcal{A}$ & $\left\{-1, Y_{*}<0\right\}$ & $e^{H_{0} t}$ & 0 & 1 & -1 & Attractor \\
\hline $\mathcal{B}$ & $\left.\frac{A}{4(A+1)},-\frac{A}{2(A+1)}\right\}$ & $t^{\frac{2(A+1)}{3 \gamma}}$ & $t^{-2(A+1)}$ & $1-\frac{A(3 A+4) G}{(A+2) \Lambda}$ & $\frac{3 A \gamma}{2(1+A)}-1$ & $\begin{array}{c}\text { Saddle for } A>8 \\
\text { Repeller (focus) for } 0<A<8\end{array}$ \\
\hline
\end{tabular}

Table III. The critical points for Model III.

Using the relation $T=-6 H^{2}$, this equation can be rewritten as

$$
\frac{f_{1}\left(H^{2}\right)+12 H^{2} f_{1}^{\prime}\left(H^{2}\right)}{f_{2}\left(H^{2}\right)+12 H^{2} f_{2}^{\prime}\left(H^{2}\right)}-\frac{\rho_{m}}{\kappa}=0 .
$$

In the above equation the first term is a function of $H^{2}$ only, and hence it could be formally solved for $\mathrm{H}^{2}$ giving

$$
H^{2}-g\left(\frac{\rho_{m}}{\kappa}\right)=0
$$

where $g\left(\rho_{m} / \kappa\right)$ a function of its argument. Relation 60 implies that this scenario is equivalent to general relativity plus a fluid with a non-trivial equation of state. Additionally, it implies that there is a certain degree of degeneracy in the cosmological application of this gravitational modification: there are multiple combinations of $f_{1}$ and $f_{2}$ that give rise to the same function $g$.

Let us now define the variables

$$
X=\frac{f_{1}-2 T f_{1}^{\prime}}{T\left(2 T f_{2}^{\prime}-f_{2}\right)}, \quad \Omega=\frac{\rho_{m}}{6 \kappa H^{2}},
$$

and the $\operatorname{logarithmic}$ time $N=\log a$. In this way Eqs. (60) tells us that $X=\Omega$, i.e. there is only one independent variable and the phase space is of dimension one. In the following we will choose $\Omega$ as a key variable due to its straightforward physical meaning.

In the same way, Eq. 12 can be written as

$$
\begin{gathered}
\dot{H}\left[\kappa\left(f_{1}^{\prime}+2 T f_{1}^{\prime \prime}\right)-\rho_{m}\left(f_{2}^{\prime}+2 T f_{2}^{\prime \prime}\right)\right] \\
+\frac{1}{4} \gamma \rho_{m}\left(f_{2}-2 T f_{2}^{\prime}\right)=0
\end{gathered}
$$

where we have used the relation $\dot{T}=-12 H \dot{H}$. In terms of the variables given in Eq. [61, , one may write Eq. 62) as

$$
X[A(T)+\Omega B(T)] \frac{\dot{H}}{H^{2}}-\frac{3 \gamma}{2} \Omega=0,
$$

where $A(T)$ and $B(T)$ are defined as

$$
\begin{aligned}
& A(T)=\frac{T\left(f_{1}^{\prime}+2 T f_{1}^{\prime \prime}\right)}{f_{1}-2 T f_{1}^{\prime}}, \\
& B(T)=\frac{T^{2}\left(f_{2}^{\prime}+2 T f_{2}^{\prime \prime}\right)}{f_{1}-2 T f_{1}^{\prime}},
\end{aligned}
$$

respectively. Now, provided that the equation

$$
\frac{f_{1}-2 T f_{1}^{\prime}}{T\left(2 T f_{2}^{\prime}-f_{2}\right)}=\Omega
$$

can be inverted, the dynamics of the full cosmological system is described solely by a single equation for $\Omega$, namely

$$
\frac{d \Omega}{d N}=-3 \gamma \Omega\left[1+\frac{1}{A(\Omega)+B(\Omega) \Omega}\right] .
$$

The stability of the fixed points of this equation can be trivially obtained considering its second derivative with respect to $\Omega$, evaluated at the fixed points. The associated solution will be given integrating

$$
\begin{aligned}
& \frac{\dot{H}}{H^{2}}=-\frac{1}{p} \\
& \frac{1}{p}=-\frac{3 \gamma}{2\left(A\left(\Omega_{*}\right)+B\left(\Omega_{*}\right) \Omega_{*}\right)},
\end{aligned}
$$

where the subscript $*$ represents the value of $\Omega$ at the fixed point. Similarly to the usual method of the previous section, integrating and using the Bianchi identities we obtain

$$
\begin{aligned}
& a=a_{0}\left(t-t_{0}\right)^{p}, \\
& \rho=\rho_{0}\left(t-t_{0}\right)^{-3 \gamma p} .
\end{aligned}
$$

Finally, from the structure of Eq. 66 we deduce that one of the possible solutions can be the vacuum $(\Omega=0)$ one, but its existence depends on the form of the second factor. A second class of points, namely $\mathcal{B}$, can arise from the term in square parenthesis in 66, representing a scaling solution with decelerated expansion.

In the following three subsections, we apply the above general method in three specific examples based on different choices of $f_{1}$ and $f_{2}$.

\section{A. Example 1}

Let us consider the functions

$$
f_{1}=-\frac{\alpha}{9} T^{5}, \quad f_{2}=-\frac{\beta}{3} T
$$


where $\alpha, \beta$ are constants. In this case we have

$$
T=-\sqrt{\frac{\beta \Omega}{\alpha}},
$$

and therefore Eq. (66) reads

$$
\frac{d \Omega}{d N}=2 \gamma \Omega,
$$

which admits the fixed point $\Omega=0$. The solution associated to this fixed point can be found integrating (67) at the fixed point, once the functions $A$ and $B$ have been substituted. In particular, we find

$$
\frac{\dot{H}}{H^{2}}=-\frac{1}{2} \gamma,
$$

which leads to the scaling solution

$$
a=a_{0}\left(t-t_{0}\right)^{\frac{2}{\gamma}},
$$

that corresponds to accelerated expansion. It is easy to see that this point is an attractor for all parameter values. However, since this point corresponds to $T=0$, this form of the functions $f_{1}$ and $f_{2}$ cannot be accepted as physically motivated.

\section{B. Example 2}

As a second example, let us consider the functions

$$
f_{1}=-\frac{\alpha}{6} T, \quad f_{2}=-\frac{\delta \sqrt{-T}-\eta T}{2 T},
$$

where $\alpha, \beta, \delta, \eta$ are positive constants. In this case we obtain

$$
T=-\frac{36 \delta^{2} \Omega^{2}}{(\alpha+6 \eta \Omega)^{2}},
$$

and therefore Eq. (66) becomes

$$
\frac{d \Omega}{d N}=\frac{\gamma \Omega(\alpha+6 \eta \Omega)}{\alpha-6 \eta \Omega},
$$

where without a significant loss of generality we have assumed that all constants are positive. This equation admits only one fixed points with non negative coordinates, namely $\Omega=0$. The corresponding solutions are given by

$$
a=a_{0}\left(t-t_{0}\right)^{\frac{1}{3 \gamma}}
$$

which represents a decelerated expansion scenario. This scaling solution is always unstable, and this implies the presence of asymptotic attractors. It is also interesting to note that since the (76) posses a singularity in this case, like in the examples of the previous sections, there are initial conditions for which the system evolves towards a singularity. This implies that in general the models at hand can present dynamical instabilities.

\section{Example 3}

Finally, let us consider the functions

$$
f_{1}=\log (-T)+2+\alpha, \quad f_{2}=-\frac{\beta[3 \log (-T)+2]}{9 T},
$$

where $\alpha, \beta$ are constants. In this case we find

$$
T=-\exp \left(\frac{\alpha}{\beta \Omega-1}\right),
$$

and therefore Eq. 66 reads as

$$
\frac{d \Omega}{d N}=-\frac{3 \gamma \Omega(\beta \Omega+1)^{2}}{\beta \Omega(\alpha+2+\beta \Omega)+1},
$$

which admits two non-negative fixed points, namely $\Omega=$ 0 and $\Omega=1 / \beta$. The corresponding solutions are the de-Sitter expansion and the Friedmann solution (matterdominated expansion). The first one is stable for any values of the parameters $\alpha, \beta$ and positive $w$. However, the Friedmann point is not hyperbolic: it is an attractor for $\Omega>1 / \beta$ and a repeller for $\Omega<1 / \beta$. This point will give a consistent value of the parameter $\Omega$ for $\beta>$ 1. Hence, for this specific model the transition between matter-dominated epoch and de-Sitter dark-energy era can be described naturally.

Like in the previous example, also in this case the phase space presents singular points. Some initial condition lead to these singularities showing also in this case the possibility of instabilities of this class of theories.

\section{DISCUSSIONS AND FINAL REMARKS}

Recently, a generalized $f(T)$ gravity theory with a nonminimal coupling between matter and the torsion scalar was proposed in 21]. From the physical point of view, in this theory, matter is not just a passive component in the space-time continuum; rather, it plays an active role in the overall gravitational dynamics, which is strongly modified due to the supplementary interaction between matter and geometry [21, 22, 30]. Moreover, the major advantage of the $f(T)$-type models, namely that the field equations are of second order, is not modified by the torsion-matter coupling [20].

In this work we have performed a detailed phase-space analysis for this scenario, proposing two new approaches. The first one, although in principle more general, turns out to lead to complications, not only in terms of the dynamical equations, but also in terms of external constraints that have to be considered during the dynamical analysis. Hence, we have developed a second method, which can be alternatively used in cases where the above complexities make the application of the first method difficult, since in this approach all constraints are included in the initial equations. Definitely, the physical results do not depend on the specific mathematical method used, 
and thus one can choose the easier method according to the specific problem.

We have analyzed six specific models of non-minimally matter-torsion coupled cosmology. In general we have extracted fixed points corresponding either to dark-matter dominated, scaling decelerated solutions, or to darkenergy dominated accelerated solutions. Additionally, note that there is a small parameter region in which the model can experience the transition from the matter epoch to dark-energy era. This feature is in agreement with the observed universe evolution, and cannot be easily obtained in dark energy models. It is also worth mentioning that in both the approaches reveal that there are sets of initial conditions for which the models evolve towards singular states for the equations. This tendency is a signal of instability related with certain specific forms of the coupling functions and this feature constraints even more the viable parameter space of these models.

We mention that in the present analysis we have focused on the finite regions of the phase space, without examining the possible non-trivial behavior at infinity, since such an extended investigation would lie beyond the scopes of this work, which is just to reveal the capabilities of the theory in producing a cosmology in agreement with observations. As we showed this is possible, and hence non-minimally matter-torsion coupled gravity may be a successful candidate for the description of Nature.

\section{ACKNOWLEDGMENTS}

SC was supported by the Fundação para a Ciência e Tecnologia through project IF/00250/2013. FSNL acknowledges financial support of the Fundação para a Ciência e Tecnologia through an Investigador FCT Research contract, with reference IF/00859/2012, and the grants EXPL/FIS-AST/1608/2013 and PEstOE/FIS/UI2751/2014. GO wishes to thank the Laboratory for High-Energy Physics (LAFEX) of the Brazilian Centre for Research in Physics, CBPF, for hospitality, PCI/MCT-CNPq and CAPES (PNPD) for financial support.
[1] S. Perlmutter, et al., Astrophys. J. 517 (1999) 565; A. G. Riess, et al., Astron. J. 116 (1998) 1009.

[2] D. N. Spergel, et al., Astrophys. J. Suppl. Ser. 148 (2003) 175; D. N. Spergel, et al., Astrophys. J. Suppl. Ser. 170 (2007) 377; E. Komatsu, et al., Astrophys. J. Suppl. Ser. 180 (2009) 330; E. Komatsu, et al., Astrophys. J. Suppl. Ser. 192 (2011) 18.

[3] M. Tegmark, et al., Phys. Rev. D 69 (2004) 103501; U. Seljak, et al., Phys. Rev. D 71 (2005) 103515.

[4] D. J. Eisenstein, et al., Astrophys. J. 633 (2005) 560.

[5] B. Jain and A. Taylor, Phys. Rev. Lett. 91 (2003) 141302.

[6] E. J. Copeland, M. Sami and S. Tsujikawa, Int. J. Mod. Phys. D 15, 1753 (2006).

[7] Y. F. Cai, E. N. Saridakis, M. R. Setare and J. Q. Xia, Phys. Rept. 493, 1 (2010).

[8] S. Nojiri and S. D. Odintsov, eConf C0602061, 06 (2006), Int. J. Geom. Meth. Mod. Phys. 4, 115 (2007).

[9] S. Capozziello and M. De Laurentis, Phys. Rept. 509, 167 (2011).

[10] C. Möller, Mat. Fys. Skr. Dan. Vid. Selsk. 1, 10 (1961); C. Pellegrini and J. Plebanski, Mat. Fys. Skr. Dan. Vid. Selsk. 2, 4 (1963); K. Hayashi and T. Shirafuji, Phys. Rev. D 19, 3524 (1979); Addendum-ibid. 24, 3312 (1982).

[11] R. Aldrovandi and J. G. Pereira, Teleparallel Gravity: An Introduction (Springer, Dordrecht, 2013); J. G. Pereira, Teleparallelism: a new insight into gravitation, in Springer Handbook of Spacetime, ed. by A. Ashtekar and V. Petkov.

[12] J. G. Pereira, AIP Conf. Proc. 1483, 239 (2012).

[13] J. W. Maluf, J. Math. Phys. 35 (1994) 335; H. I. Arcos and J. G. Pereira, Int. J. Mod. Phys. D 13, 2193 (2004).

[14] R. Ferraro and F. Fiorini, Phys. Rev. D 75, 084031 (2007); R. Ferraro, F. Fiorini, Phys. Rev. D78, 124019 (2008).
[15] E. V. Linder, Phys. Rev. D 81, 127301 (2010); Erratum, Phys. Rev. D, 82, 109902.

[16] S. H. Chen, J. B. Dent, S. Dutta and E. N. Saridakis, Phys. Rev. D 83, 023508 (2011); P. Wu, H. W. Yu, Phys. Lett. B693, 415 (2010); G. R. Bengochea, Phys. Lett. B695, 405 (2011); J. B. Dent, S. Dutta, E. N. Saridakis, JCAP 1101, 009 (2011); R. J. Yang, Europhys. Lett. 93, 60001 (2011); R. Zheng and Q. G. Huang, JCAP 1103, 002 (2011); K. Bamba, C. Q. Geng, C. C. Lee and L. W. Luo, JCAP 1101, 021 (2011); Y. -F. Cai, S. H. Chen, J. B. Dent, S. Dutta, E. N. Saridakis, Class. Quant. Grav. 28, 215011 (2011); M. Sharif, S. Rani, Mod. Phys. Lett. A26, 1657 (2011); M. Li, R. X. Miao and Y. G. Miao, JHEP 1107, 108 (2011); S. Capozziello, V. F. Cardone, H. Farajollahi and A. Ravanpak, Phys. Rev. D 84, 043527 (2011); M. H. Daouda, M. E. Rodrigues and M. J. S. Houndjo, Eur. Phys. J. C 72, 1890 (2012); K. Bamba and C. -Q. Geng, JCAP 1111, 008 (2011); Y. P. Wu and C. Q. Geng, Phys. Rev. D 86, 104058 (2012); C. G. Bohmer, T. Harko and F. S. N. Lobo, Phys. Rev. D 85, 044033 (2012); H. Wei, X. J. Guo and L. F. Wang, Phys. Lett. B 707, 298 (2012); K. Atazadeh and F. Darabi, Eur.Phys.J. C72 (2012) 2016; H. Farajollahi, A. Ravanpak and P. Wu, Astrophys. Space Sci. 338, 23 (2012); K. Karami and A. Abdolmaleki, JCAP 1204 (2012) 007; W. -S. Zhang, C. Cheng, Q. -G. Huang, M. Li, S. Li, X. -D. Li and S. Wang, Sci. China Phys. Mech. Astron. 55, 2244 (2012); L. Iorio and E. N. Saridakis, Mon. Not. Roy. Astron. Soc. 427, 1555 (2012); M. Zubair, and S. Waheed, Astrophys. Space Sci. (2015) 360:68.

[17] N. Tamanini and C. G. Boehmer, Phys. Rev. D 86, 044009 (2012); V. F. Cardone, N. Radicella and S. Camera, Phys. Rev. D 85, 124007 (2012); A. Aviles, A. Bravetti, S. Capozziello and O. Luongo, Phys. Rev. 
D 87, 064025 (2013); Y. C. Ong, K. Izumi, J. M. Nester and P. Chen, Phys. Rev. D 88 (2013) 2, 024019; H. Dong, J. Wang and X. Meng, Eur. Phys. J. C 73, no. 8, 2543 (2013); J. Amoros, J. de Haro and S. D. Odintsov, Phys. Rev. D 87, 104037 (2013); D. Liu and M. J. Reboucas, Phys. Rev. D 86, 083515 (2012); S. Chattopadhyay and A. Pasqua, Astrophys. Space Sci. 344, 269 (2013); K. Bamba, J. de Haro and S. D. Odintsov, JCAP 1302, 008 (2013); M. Jamil, D. Momeni and R. Myrzakulov, Eur. Phys. J. C 72, 2267 (2012); A. V. Astashenok, Astrophys. Space Sci. 351, 377 (2014); S. Nesseris, S. Basilakos, E. N. Saridakis and L. Perivolaropoulos, Phys. Rev. D 88, 103010 (2013); J. -T. Li, C. -C. Lee and C. Q. Geng, Eur. Phys. J. C 73, 2315 (2013); K. Bamba, S. Capozziello, M. De Laurentis, S. 'i. Nojiri and D. SezGmez, Phys. Lett. B 727, 194 (2013); G. Kofinas and E. N. Saridakis, Phys. Rev. D 90, 084044 (2014); W. El Hanafy and G. G. L. Nashed, Eur. Phys. J. C 75, 279 (2015); S. Capozziello, O. Luongo and E. N. Saridakis, Phys. Rev. D 91, no. 12, 124037 (2015) arXiv:1503.02832 [gr-qc]]; M. Krššák and E. N. Saridakis, arXiv:1510.08432 $[\mathrm{gr}-\mathrm{qc}]$.

[18] C. -Q. Geng, C. -C. Lee, E. N. Saridakis, Y. -P. Wu, Phys. Lett. B704, 384 (2011); C. Xu, E. N. Saridakis and G. Leon, JCAP 1207, 005 (2012); A. Banijamali and B. Fazlpour, Astrophys. Space Sci. 342, 229 (2012); G. Otalora, JCAP 1307, 044 (2013); G. Otalora, Phys. Rev. D 88, 063505 (2013); C. -Q. Geng, J. -A. Gu and C. -C. Lee, Phys. Rev. D 88, 024030 (2013); G. Otalora, Int. J. Mod. Phys. D 25, 1650025 (2016); H. M. Sadjadi, arXiv:1510.02085 [gr-qc]; M. Zubair, and S. Waheed, Astrophys. Space Sci. (2015) 355:361?369.

[19] T. Wang, Phys. Rev. D84, 024042 (2011); R. -X. Miao, M. Li and Y. -G. Miao, JCAP 1111, 033 (2011); C. G. Boehmer, A. Mussa and N. Tamanini, Class. Quant. Grav. 28, 245020 (2011); M. Hamani Daouda, M. E. Rodrigues and M. J. S. Houndjo, Eur. Phys. J. C 71, 1817 (2011); R. Ferraro, F. Fiorini, Phys. Rev. D 84, 083518 (2011); M. H. Daouda, M. E. Rodrigues and M. J. S. Houndjo, Eur. Phys. J. C 72, 1890 (2012); P. A. Gonzalez, E. N. Saridakis and Y. Vasquez, JHEP 1207, 053 (2012); S. Capozziello, P. A. Gonzalez,
E. N. Saridakis and Y. Vasquez, JHEP 1302 (2013) 039; K. Atazadeh and M. Mousavi, Eur. Phys. J. C 72, 2272 (2012).

[20] Y. F. Cai, S. Capozziello, M. De Laurentis and E. N. Saridakis, arXiv:1511.07586 [gr-qc].

[21] T. Harko, F. S. N. Lobo, G. Otalora and E. N. Saridakis, Phys. Rev. D 89, 124036 (2014).

[22] O. Bertolami, C. G. Boehmer, T. Harko and F. S. N. Lobo, Phys. Rev. D 75, 104016 (2007); T. Harko, T. S. Koivisto and F. S. N. Lobo, Mod. Phys. Lett. A 26, 1467 (2011).

[23] T. Harko, F. S. N. Lobo, G. Otalora and E. N. Saridakis, JCAP 1412, no. 12, 021 (2014).

[24] T. Harko, F. S. N. Lobo, S. Nojiri and S. D. Odintsov, Phys. Rev. D 84, 024020 (2011).

[25] Wainwright J and Ellis G F R (ed) 1997 Dynamical systems in cosmology Cambridge: Cambridge University Press (see also references therein).

[26] S. Carloni, P. K. S. Dunsby, S. Capozziello and A. Troisi, Class. Quant. Grav. 22 (2005) 4839; J. A. Leach, S. Carloni and P. K. S. Dunsby, Class. Quant. Grav. 23 (2006) 4915; S. Carloni and P. K. S. Dunsby, J. Phys. A 40 (2007) 6919; M. Abdelwahab, S. Carloni and P K. S. Dunsby, Class. Quant. Grav. 25 (2008) 135002; S. Carloni, A. Troisi and P. K. S. Dunsby, Gen. Rel. Grav. 41 (2009) 1757; X. Roy, T. Buchert, S. Carloni and N. Obadia, Class. Quant. Grav. 28 (2011) 165004; A. Bonanno and S. Carloni, New J. Phys. 14 (2012) 025008; G. Leon and E. N. Saridakis, JCAP 1303, 025 (2013); S. Carloni, S. Vignolo and L. Fabbri, Class. Quant. Grav. 30 (2013) 205010; S. Carloni, JCAP 1509, no. 09, 013 (2015); S. Carloni, T. Koivisto and F. S. N. Lobo, Phys. Rev. D 92, no. 6, 064035 (2015).

[27] O. Groen and S. Hervik, Einstein's general theory of relativity, New York, USA: Springer (2007).

[28] O. Bertolami, F. S. N. Lobo and J. Paramos, Phys. Rev. D 78, 064036 (2008) doi:10.1103/PhysRevD.78.064036 arXiv:0806.4434 [gr-qc]].

[29] M. M. Guterman and Z. H. Nitecki, Differential Equations: A First Course, 3rd ed., Saunders (1992).

[30] T. Harko, Phys. Lett. B 669, 376 (2008). 